\title{
Desempenho dos médicos na saúde da família - uma análise a partir dos princípios ordenadores em um município brasileiro
}

\author{
Viviane Aparecida Alvares da Silva \\ Universidade Federal de Minas Gerais / Faculdade de Ciências Econômicas \\ Belo Horizonte / MG - Brasil \\ Allan Claudius Queiroz Barbosa \\ Universidade Federal de Minas Gerais / Faculdade de Ciências Econômicas \\ Belo Horizonte / MG - Brasil \\ Thiago Augusto Hernandes Rocha \\ Universidade Federal de Minas Gerais / Faculdade de Ciências Econômicas, \\ Centro de Pós-graduação e Pesquisas em Administração \\ Belo Horizonte / MG - Brasil
}

\begin{abstract}
A partir dos princípios ordenadores da atenção primária (AP), definidos por Starfield, o presente artigo analisa em que medida o desempenho dos médicos da Estratégia Saúde da Família (ESF) contribui para a consolidação de tais princípios. Trata-se de um estudo descritivo, no qual foram utilizados indicadores de desempenho, desdobrados a partir dos seis princípios ordenadores da ESF, a fim de mensurar o desempenho destes profissionais no município de Belo Horizonte. Os resultados encontrados, em geral, indicaram que o desempenho do médico da família favorece o cumprimento dos princípios ordenadores. Consoante com a literatura, os resultados da análise de regressão múltipla demonstraram que o desempenho da equipe de saúde influencia o desempenho do médico, o que reforça a importância da adoção de modelos de avaliação de performance médica que considerem múltiplos determinantes.
\end{abstract}

Palavras-chave: desempenho; estratégia saúde da família; gestão de recursos humanos em saúde; médico.

Rendimiento de los médicos en la salud de la familia — un análisis de los principios ordenadores en una ciudad brasileña

A partir de los principios ordenadores de la atención primaria (AP) definido por Starfield, este artículo analiza el grado en que el desempeño de los médicos de la Estrategia Salud de la Familia (ESF) contri-

DOI: http://dx.doi.org/10.1590/0034-7612140407

Artigo recebido em 10 set. 2014 e aceito em 30 abr. 2015. 
buye a la consolidación de estos principios. Se trata de un estudio descriptivo en el que se utilizaron los indicadores de desempeño, desplegado a partir de seis principios ordenadores del ESF con el fin de medir el desempeño de estos profesionales en la ciudad de Belo Horizonte. Los resultados indican generalmente que el desempeño del médico de familia favorece el cumplimiento de los principios ordenadores. De acuerdo con la literatura, los resultados del análisis de regresión múltiple mostraron que el rendimiento del equipo de salud influye en el desempeño del médico, lo que refuerza la importancia de la adopción de modelos de evaluación de desempeño médica considerando múltiples factores determinantes.

Palabras clave: rendimiento; estrategia de salud familiar; gestión de recursos humanos en salud; médico.

Performance of physicians in family health — an analysis from the guiding principles in a Brazilian municipality

From the guiding principles of primary care (AP), defined by Starfield, this article analyzes the extent to which performance of the doctors of the Family Health Strategy (ESF) contributes to the consolidation of these principles. For this purpose, it was carried out a descriptive study in which performance indicators were used, deployed from six guiding principles of the ESF, in order to measure the performance of these professionals in the city of Belo Horizonte. The results generally indicated that the performance of the family's doctor underpins the achievement of the guiding principles. According to the literature, the results of multiple regression analysis showed that the health team's performance influences the doctor's performance, which reinforces the importance of adopting medical performance evaluation models that consider multiple determinants.

KEYWORDS: performance; family health; management of human resources for health; physician.

\section{Apresentação}

O uso recente de indicadores e sistemas de avaliação de desempenho tem se fortalecido em diversos campos e com a oferta de serviços de saúde não é diferente (Exworthy et al., 2003). Essa tendência se observa em decorrência de pressões originárias dos formuladores de políticas, bem como dos pacientes, almejando organizações de saúde preocupadas com a eficiência e a melhora do cuidado ofertado (Marshall, Haywood e Fitzpatrick, 2006). A saúde conta com um regime de mão de obra intensivo, o que torna o acompanhamento sistemático do desempenho dos profissionais um imperativo, tendo como premissa que o desempenho dos recursos humanos tem o potencial de influenciar os resultados obtidos pela oferta de serviços (Rocha et al., 2014).

Tendo por pano de fundo o contexto da Estratégia Saúde da Família (ESF), o presente trabalho busca discutir o desempenho dos médicos que atuam na ESF, considerando o cumprimento de suas atribuições quanto aos princípios ordenadores que regem seu funcionamento. Para tanto, foram utilizados indicadores de desempenho, a fim de mensurar a performance dos médicos no município de Belo Horizonte. Além disso, este estudo buscou averiguar se a performance do médico sofre influência das variáveis: desempenho da equipe de Saúde da Família (SF), infraestrutura da Unidade Básica de Saúde (UBS), tempo de permanência do médico na equipe de $\mathrm{SF}$ e formação profissional. 


\section{A atenção primária à saúde no Sistema Único de Saúde (SUS)}

\subsection{A ESF como eixo estruturador do SUS}

O SUS foi implantado em 1988, e regulamentado pelas Leis Orgânicas da Saúde no 8.080 e no 8.142 , em 1990, as quais alteraram a situação de desigualdade observada na assistência à saúde da população brasileira (Brasil, 1990; Mendes, 2002). Essas leis estabeleceram as diretrizes do sistema de saúde: universalidade de acesso, integralidade de assistência e equidade (Silva, 2009).

A fim de superar as dificuldades iniciais e buscando colocar em prática as diretrizes do SUS, o Ministério da Saúde (MS) implantou em 1994 o Programa Saúde da Família, posteriormente denominado "Estratégia Saúde da Família" (Mendes, 2002). A Estratégia Saúde da Família é caracterizada por desenvolver atividades de promoção à saúde, prevenção de agravos e oferta de cuidado de nível primário, ofertando atenção contínua nas especialidades básicas por intermédio de uma equipe multiprofissional (Brasil, 1997), composta por médico, enfermeiro, auxiliares de enfermagem, bem como agentes comunitários de saúde (Mendes, 2002). Os serviços de saúde organizados a partir da ESF estão apoiados em um conjunto de princípios denominados "ordenadores" (quadro 1), que conduzem o funcionamento deste modelo de atenção à saúde (Starfield, 2002; Mendes, 2002).

\section{Quadro 1}

\section{Princípios ordenadores da saúde da família}

\begin{tabular}{|ll|}
\hline Princípio & Descrição \\
\hline Primeiro Contato & $\begin{array}{l}\text { Refere-se ao acesso e uso do serviço de saúde a cada novo problema pelo qual as pessoas procuram } \\
\text { pela atenção à saúde. Um serviço pode ser considerado porta de entrada quando a população e a } \\
\text { equipe o identificam como primeiro recurso de saúde. }\end{array}$ \\
Longitudinalidade & $\begin{array}{l}\text { Aporte de cuidados prestados pela equipe de saúde ao longo do tempo, num ambiente de relação } \\
\text { mútua e humanizada entre equipe de saúde, indivíduos e famílias. }\end{array}$ \\
Integralidade & $\begin{array}{l}\text { Prestação de todos os tipos de serviços de saúde que atendam às necessidades da população adscrita } \\
\text { para o manejo definitivo de problemas específicos. }\end{array}$ \\
Foco na Família & $\begin{array}{l}\text { Integração dos níveis primário, secundário e terciário. Capacidade de garantir a continuidade da atenção } \\
\text { com o reconhecimento dos problemas que requerem seguimento constante. } \\
\text { Onteraño da equipe de saúde com essa unidade social e o conhecimento integral de seus problemas } \\
\text { Reconhecimento das necessidades familiares em função do contexto físico, social e cultural em que } \\
\text { vivem os pacientes. O reconhecimento do contexto social e o entendimento das condiçães de vida } \\
\text { do paciente são relevantes, já que todos os problemas de saúde ocorrem dentro de um ambiente } \\
\text { social que frequentemente predispõe ou causa enfermidades. }\end{array}$ \\
\hline
\end{tabular}

Fonte: Adaptado de Starfield (2002) e Mendes (2002). 
A partir dos esforços observados para a implantação e expansão da ESF e perante os desafios que se colocam para sua operacionalização, faz-se necessário refletir sobre o profissional médico, tendo em vista sua importância.

\subsection{0 profissional médico e sua inserção na ESF}

O médico exerce um papel de protagonismo na avaliação da demanda do paciente e dos riscos individuais de adoecer, bem como na elaboração de um projeto terapêutico (Capozzolo, 2003). Na ESF, o médico deve procurar compreender a doença em seu contexto pessoal, familiar e social e se integrar ao ambiente local, de forma a ofertar cuidados modelados às necessidades da comunidade adscrita (Brasil, 2012). Assim, deve-se dedicar uma atenção especial a esse profissional, por parte dos gestores em saúde, uma vez que o mesmo é essencial para o funcionamento dos serviços, sendo considerado o ator responsável em procedimentos como consultas regulares, prescrições de medicamento e encaminhamento para outros níveis de atenção (Rodrigues et al., 2011). No que tange à formação do profissional, faz-se necessário um perfil generalista para atender as famílias na ESF, oferecendo atenção integral, abordando aspectos de prevenção e educação sanitária e empenhando-se para manter seus pacientes saudáveis.

Diante dessa relevância, no que tange à oferta de serviços dedicados à atenção primária, a abordagem de aspectos que tangenciem o desempenho do médico se confunde com o exame da qualidade dos serviços prestados pelas equipes da ESF. Em função dessa associação, há um crescente aumento de interesse internacional no monitoramento e avaliação do desempenho profissional de médicos (Wright et al., 2012). Esse interesse tem sido marcado pela multiplicidade de abordagens utilizadas para se avaliar o desempenho de médicos (De Vries et al., 2009); apesar disso, não foram reportadas iniciativas nesse sentido, adaptadas ao contexto brasileiro. Assim, faz-se necessário caracterizar o que se depreende por desempenho, considerando-se os médicos na ESF.

\subsection{Desempenho médico e a temática de recursos humanos em saúde}

Diversos países têm engendrado esforços com o intuito de suplantar essas dificuldades e contribuir para a consolidação de serviços de saúde de qualidade. A situação do Brasil não é diferente, mas podem-se destacar algumas especificidades, decorrentes do contexto brasileiro. Entre tais dificuldades destacam-se: exigências sindicais de flexibilização de regras, atração e fixação de profissionais em áreas remotas, estruturação de uma carreira nacional de saúde, precarização de vínculos de trabalhos, multiplicidade de vínculos simultâneos, influências políticas na contratação e demissão de profissionais, altos índices de absenteísmo e necessidade de adaptação e alinhamento de políticas de formação e atualização profissional (Buchan et al., 2011). 
Os desafios colocados afetam a ESF brasileira, limitando as possibilidades de melhorias. Grace e colaboradores (2014) chamam a atenção para o fato de que esse tipo de situação ocorre com frequência junto à atenção primária à saúde, uma vez que, junto a um estudo desenvolvido no Canadá, constataram que 15\% dos médicos de família atuavam com consideráveis deficiências em termos de atuação e formação.

Assegurar qualidade quanto à prática médica é um objetivo a ser perseguido pelos sistemas de saúde. Programas que busquem avaliar o desempenho de profissionais médicos são ferramentas úteis na consecução de tais objetivos, uma vez que permitem identificar aqueles profissionais que carecem de intervenções para a melhoria de formação.

Sistemas de avaliação de desempenho, usualmente, se valem de medidas discretas ou de resultados clínicos para poder definir patamares comparativos entre o padrão auferido pelos profissionais e aquele nível de desempenho que seria o desejável. Assim, a mensuração de desempenho figura como um elemento de destaque, particularmente quando as métricas desenvolvidas são compartilhadas com a equipe clínica com o intuito de viabilizar a identificação de estratégias para a promoção de melhorias (Cassel e Jain, 2012).

A performance dos indivíduos no trabalho depende de múltiplos fatores (Oliveira-Castro, Lima e Veiga, 1996; Sonnentag e Frese, 2002; Abbad et al., 2006). O desempenho dos profissionais resulta não apenas das competências individuais, mas também das relações interpessoais, do ambiente de trabalho e das características da organização (Brandão e Guimarães, 2001). Os fatores motivacionais, também, interferem no desempenho dos profissionais (Abbad et al., 2006).

O entendimento dos fatores que dão suporte ao bom desempenho médico é essencial para a manutenção da segurança dos pacientes e proteção do interesse público (Grace et al., 2014). O desempenho profissional do médico é o resultado da articulação de diversos elementos como características pessoais, tais quais: idade, gênero, especialidade, estado de saúde, formação, bem como do contexto, no qual sua prática se dá (Grace et al., 2014). Quanto a este último aspecto, exercem influência sobre os níveis de desempenho: a estrutura física para a oferta do cuidado, a comunidade em que se insere o profissional, os padrões de remuneração e as perspectivas de carreira, por exemplo (Grol, 2002; Hogg et al., 2008; Russell et al., 2009).

O caráter multideterminado do desempenho médico determina a relevância de se realizar avaliações num contexto ambiental mais amplo, de maneira a não circunscrever sua determinação apenas às características pessoais do profissional. Modelos que busquem avaliar o desfecho do cuidado ofertado pelo profissional médico devem considerá-lo um construto complexo, resultante de efeitos combinados, mais que um simples reflexo de ações de treinamento e credenciais de formação (Grace et al., 2014).

O processo de avaliação de desempenho deve ser capaz de: valorizar o desempenho eficaz e identificar as discrepâncias na performance dos profissionais; identificar e remover os obstáculos e as restrições organizacionais ao desempenho; apoiar a tomada de decisão quanto à carreira dos profissionais e promover e aperfeiçoar programas e políticas de desenvolvimento de recursos humanos (Oliveira-Castro, Lima e Veiga, 1996). 
A carência de trabalhos que abordem a temática referente à avaliação de desempenho do médico suscita a necessidade de adaptar o que é discutido na literatura internacional para a realidade brasileira, de modo a produzir evidências que auxiliem na consecução de melhorias junto ao SUS. O setor de saúde é carente de estudos avaliativos com foco em gestão que procurem estabelecer parâmetros de relacionamento entre práticas de GRH e o cumprimento de oferta de cuidado. Nesse sentido, a ESF é mais carente ainda, uma vez que não foram encontrados na literatura estudos, adaptados à realidade brasileira, que investigassem aspectos atrelados ao desempenho do médico e à eficiência na execução de ações de oferta de cuidado, a qual pode ser considerada uma vertente do desempenho.

Assim, o presente trabalho buscou identificar de que maneira os diferentes padrões de desempenho do médico se relacionam às medidas de cumprimento de processo de trabalho em saúde na esfera da ESF. Para tanto, foram desenvolvidos indicadores voltados para a avaliação do desempenho do médico, bem como medidas de eficiência no cumprimento de ações adscritas ao escopo da ESF. Uma vez definidas tais medidas, o relacionamento entre os aspectos de estrutura e processo foi analisado com ênfase na performance do médico, de modo a possibilitar a análise de parâmetros que podem modular a qualidade da oferta de cuidado.

\section{Procedimentos metodológicos}

\subsection{Caracterização da pesquisa}

Para abordar o objeto em questão optou-se pela pesquisa quantitativa. Segundo Roesch (2010), a pesquisa quantitativa é recomendada nos casos em que o propósito do projeto implica medir relações entre variáveis. Gonçalves e Meirelles (2004) pontuam que os dados nesse tipo de pesquisa são representados por métricas quantitativas. O emprego da pesquisa quantitativa contribui para gerar medidas consistentes sobre o desempenho dos médicos da Saúde da Família. Além disso, esse tipo de pesquisa permite verificar se há relação entre a performance do médico e as variáveis: desempenho da equipe de Saúde da Família, infraestrutura da UBS, formação profissional e tempo de permanência do médico na equipe de SF.

Quanto a seu caráter, esta pesquisa é classificada como descritiva. Selltiz e colaboradores (1965) salientam que os estudos descritivos representam com exatidão as características de um indivíduo, situação ou grupo. Gil (2006) destaca que, além de descrever as características de uma população ou fenômeno, a pesquisa descritiva estabelece relações entre as variáveis.

Como estratégia de pesquisa, optou-se por utilizar o estudo de caso. Conforme pontua Yin (2005), o estudo de caso é uma estratégia que busca examinar o fenômeno contemporâneo em seu contexto. Segundo Goldenberg (2002), o estudo de caso considera a unidade social como um todo, seja um indivíduo, uma instituição ou uma comunidade para compreendê-los.

O município de Belo Horizonte foi escolhido como a unidade de análise. Tal escolha foi intencional, tendo em vista a possibilidade de trabalhar com dados censitários coletados sobre 
o desempenho das equipes de SF da capital mineira, o que permitiu verificar o desempenho dos médicos, utilizando para isso os indicadores referentes às atribuições deste profissional.

\subsection{Contexto e universo da pesquisa}

A ESF de Belo Horizonte conta com um aparato tecnológico de apoio local que está ancorado em redes superpostas de atenção especializada, de urgência, de saúde mental, de saúde bucal, de atenção hospitalar, de apoio diagnóstico e com articulação transversal com as ações coletivas e de promoção e prevenção de agravos (Magalhães Júnior, 2010).

Outro ponto relevante para a expansão da ESF, em Belo Horizonte, diz respeito à territorialização da saúde. Conforme pontuam Teixeira e colaboradores (2011), a distritalização da saúde foi adotada em Belo Horizonte, em 1989, voltada para construção do seu modelo de atenção à saúde. Assim, o município de Belo Horizonte é dividido em nove distritos sanitários - Barreiro, Centro Sul, Leste, Nordeste, Noroeste, Norte, Oeste, Pampulha e Venda Nova o que favorece o planejamento e a gestão do sistema de saúde (Rocha, 2011). Conforme observam Starfield (2002) e Teixeira e colaboradores (2011), é de suma importância considerar o ambiente em que vivem as pessoas para planejar as ações de saúde. O universo considerado para a realização deste estudo foram todos os médicos que atuam nas equipes de saúde da família de Belo Horizonte.

\subsection{Coleta de dados}

Para o levantamento das informações referentes ao desempenho do profissional médico da Saúde da Família, foram utilizados dados secundários provenientes do banco de dados da pesquisa "Monitoramento de resultados e desempenho e satisfação dos usuários da estratégia saúde da família: um estudo em Belo Horizonte". Optou-se por trabalhar com dados secundários em função da possibilidade de contar com os dados dos médicos de todas as equipes da Saúde da Família do município de Belo Horizonte coletados em 2010.

Graças à utilização intensiva de mão de obra, a GRH assume posição de destaque no que tange ao cumprimento dos princípios ordenadores. Problemas de GRH podem atuar como empecilhos para o efetivo cumprimento dos princípios ordenadores de uma AP forte. Starfield (2002) afirma que, para que a AP seja eficaz em promover melhores resultados em saúde, é de fundamental importância que todos os princípios ordenadores sejam efetivamente cumpridos. Os desafios associados à atração e retenção de profissionais, remuneração, multiplicidade de modalidades contratuais, ausência de uma carreira estruturada, disputa por profissionais, alta rotatividade e absenteísmo, déficits de formação e dificuldades atreladas à compreensão do novo processo de trabalho são elementos que modulam diretamente o cumprimento dos princípios ordenadores (Pustai, 2004). 
As práticas de GRH repercutem na execução do processo de trabalho relacionado aos princípios ordenadores da AP. O exemplo da rotatividade deixa claro como a dimensão de GRH pode impedir o efetivo cumprimento dos princípios ordenadores. Altos índices de rotatividade impedem a formação de um vínculo sólido entre o profissional de saúde e a comunidade na qual o mesmo se insere. A ausência de vínculo interfere no princípio da longitudinalidade, uma vez que impede um cuidado regular ao longo do tempo, assim como nos princípios da integralidade e orientação comunitária. Naquele, por influência negativa na confiança estabelecida para com o profissional de saúde, fator importante, uma vez que o vínculo entre o usuário e o profissional exerce influência na adesão ao tratamento, por exemplo (Starfield, 2002); neste, por impedir o conhecimento aprofundado das necessidades de saúde da comunidade, na qual o profissional atua, impedindo a adequação da oferta do cuidado, em função das necessidades locais. Déficits de treinamento e de formação de profissionais podem impedir a realização de atividades voltadas para o conhecimento da comunidade e diminuir a qualidade dos atendimentos prestados aos diferentes agravos.

Levando-se em conta essas especificidades, foram coletados, no âmbito deste trabalho, dados referentes ao processo de trabalho - atendimento clínico, procedimentos e campanhas de promoção e prevenção de saúde - e dados referentes a: estrutura física, disponibilidade de insumos, materiais e equipamentos nas UBS. Além disso, foram coletadas informações sobre recursos humanos, tais como: composição da equipe, carga horária de trabalho, treinamento, tempo de permanência do profissional na equipe, tipo de vínculo e formação profissional. A vinculação entre os princípios ordenadores e os indicadores de desempenho do médico é delineada no quadro 2 .

Quadro 2

Vinculação entre os princípios ordenadores e os indicadores de monitoramento de desempenho médico

\begin{tabular}{|c|c|c|}
\hline $\begin{array}{l}\text { Princípios } \\
\text { ordenadores }\end{array}$ & $\begin{array}{l}\text { Parâmetros para definição dos indicadores } \\
\text { de desempenho do médico }\end{array}$ & Indicadores de desempenho médico \\
\hline Primeiro Contato & v Realizam visitas domiciliares mensais. & \multirow{3}{*}{$\begin{array}{l}\text { - Percentual de médicos que realizam visitas } \\
\text { - Pomiciliares mensais } \\
\text { Percentual de médicos que realizam visitas } \\
\text { - Percentiares a acamados de médicos que realizam visitas } \\
\text { domiciliares e usuários com mobilidade reduzida } \\
\text { - Percentual de médicos que realizam visitas } \\
\text { domiciliares a mulher no período de puerpério } \\
\text { - Percentual de médicos que realizam visitas } \\
\text { domiciliares a população em geral (exceção: } \\
\text { acamados, usuários com mobilidade reduzida, mulher } \\
\text { no período de puerpério) } \\
\text { - Percentual de médicos que participam de reuniões } \\
\text { com a comunidade }\end{array}$} \\
\hline Foco na Família & $\begin{array}{l}\text { Vealizam visitas domiciliares a grupos } \\
\text { de usuários (acamados, usuários com } \\
\text { mobilidade reduzida, mulheres em período } \\
\text { de puerpério e população em geral). }\end{array}$ & \\
\hline $\begin{array}{l}\text { Orientação } \\
\text { Comunitária }\end{array}$ & v Realizam reuniões com a comunidade. & \\
\hline
\end{tabular}




\begin{tabular}{|c|c|c|}
\hline $\begin{array}{l}\text { Princípios } \\
\text { ordenadores }\end{array}$ & $\begin{array}{l}\text { Parâmetros para definição dos indicadores } \\
\text { de desempenho do médico }\end{array}$ & Indicadores de desempenho médico \\
\hline $\begin{array}{l}\text { Integralidade } \\
\text { Longitudinalidade } \\
\text { Coordenação }\end{array}$ & $\begin{array}{l}\text { - Cumprimento de aç̃̃es e procedimento } \\
\text { de consultas típicas relativas aos grupos: } \\
\text { saúde da mulher, saúde da criança, } \\
\text { usuários portadores de doenças crônicas } \\
\text { não transmissíveis (hipertensão arterial } \\
\text { e diabetes), saúde mental, saúde do } \\
\text { idoso e saúde do adolescente. Inclui } \\
\text { encaminhamento para outros níveis da } \\
\text { atenção e solicitação de consultas de } \\
\text { retorno, quando necessário. }\end{array}$ & $\begin{array}{l}\text { - Percentual de cumprimento das ações médicas } \\
\text { - Pelativas à saúde da mulher } \\
\text { relativas à saúde da gestante } \\
\text { - Percentual de cumprimento de ações realizadas pelo } \\
\text { médico em consulta típica de pré-natal } \\
\text { - Percentual de cumprimento das ações médicas } \\
\text { relativas à saúde da criança } \\
\text { - Percentual de cumprimento das ações médicas } \\
\text { relativas aos portadores de hipertensão arterial } \\
\text { - Percentual de cumprimento das ações médicas } \\
\text { relativas aos portadores de diabetes } \\
\text { - Percentual de cumprimento de procedimentos } \\
\text { realizados na consulta típica de doenças crônicas } \\
\text { - Percentual de cumprimento das ações médicas } \\
\text { relativas à saúde mental } \\
\text { - Percentual de cumprimento das ações médicas } \\
\text { relativas à saúde do idoso } \\
\text { - Percentual de cumprimento das ações médicas } \\
\text { relativas à saúde do adolescente }\end{array}$ \\
\hline
\end{tabular}

Fonte: Elaborada pelos autores.

Além do detalhamento das variáveis que foram levadas em conta para fins de avaliação do desempenho do médico, foram consideradas na análise da performance do médico as seguintes variáveis: desempenho da equipe de SF, infraestrutura da Unidade Básica de Saúde, formação profissional e tempo de permanência do profissional na equipe de Saúde da Família. O trabalho em equipe é um dos principais pilares da Saúde da Família. Pressupõe uma relação recíproca de comunicação e interação entre os membros da equipe. Gyorgyi (2002) aponta as condições de trabalho — entendidas neste estudo como estrutura física da Unidade Básica de Saúde e disponibilidade de insumos, materiais e equipamentos - como um dos fatores que podem interferir no desempenho dos serviços. A discussão em relação à formação do médico da SF faz menção ao tipo de médico — recomendado para atuar na Saúde da Família. Starfield (2002) destaca que médicos generalistas possuem condições mais adequadas para avaliar os múltiplos e interativos determinantes da doença e da saúde.

Quanto ao tempo de trabalho do profissional, Starfield (2002) destaca que a interação entre os profissionais de saúde e os pacientes estabelece relações de longa duração, facilitando a obtenção de melhores resultados na Atenção Primária. A partir do que foi exposto, notase que é relevante investigar se as variáveis citadas anteriormente podem influenciar o desempenho do médico. Tais variáveis e os indicadores utilizados são relacionados no quadro 3. 
Quadro 3

Variáveis consideradas contextuais com potencial de influenciar o desempenho do médico

\begin{tabular}{|c|c|c|}
\hline $\begin{array}{l}\text { Variável } \\
\text { Independente }\end{array}$ & Parâmetros para definição dos indicadores & Indicador \\
\hline $\begin{array}{l}\text { Desempenho da } \\
\text { Equipe }\end{array}$ & $\begin{array}{l}\text { - Processo de trabalho da equipe - cadastramento da população; } \\
\text { registro de informações; uso de prontuário; mapa de abrangência para } \\
\text { planejamento; perfil para área de abrangência; planejamento de ações } \\
\text { e de oferta de serviços; busca ativa (hanseníase, diabetes, hipertensão, } \\
\text { gestante, HIV, tuberculose, saúde mental); acompanhamento dos } \\
\text { pacientes baseado em protocolos clínicos; divulga a agenda para } \\
\text { usuários; supervisão da equipe (técnica, clínica), discussão de casos } \\
\text { clínicos, tratamento clínico na unidade (crise convulsiva, crise de asma } \\
\text { ou broncoespasmo, crise hipertensiva, desidratação, vômitos, corte } \\
\text { com indicação de sutura, crise de hipoglicemia); mobiliza apoio social; } \\
\text { realiza busca ativa de mulheres (alteração exame cito-patológico, } \\
\text { solicitação de mamografia das que não retornam na consulta); estuda } \\
\text { a cobertura da população-alvo da sua área de abrangência referente à } \\
\text { citologia oncótica, à mamografia } \\
\text { - Ações e procedimentos realizados pelo enfermeiro - participou de } \\
\text { reunião com a comunidade no último semestre; participou de reunião } \\
\text { de equipe nos últimos dois meses; realizou atividade educativa em } \\
\text { grupo nos últimos dois meses; realizou atividade de promoção e } \\
\text { prevenção nos últimos dois meses; realiza visitas domiciliares mensais, } \\
\text { realiza consulta de enfermagem de pré-natal programada; realiza } \\
\text { treinamento do auxiliar de enfermagem, do Acs; realiza consulta de } \\
\text { enfermagem em clínica médica, em pediatria, em ginecologia, em } \\
\text { obstetrícia } \\
\text { adolescente; à saúde do idoso }\end{array}$ & $\begin{array}{l}\text { Total de atribuições e } \\
\text { procedimentos realizados pela } \\
\text { equipe de saúde da família }\end{array}$ \\
\hline Infraestrutura & $\begin{array}{l}\text { - Disponibilidade de materiais e equipamentos; insumos; medicamentos } \\
\text { para atendimento de urgência na Unidade Básica de Saúde. Adequação } \\
\text { das instalações (acessibilidade) }\end{array}$ & $\begin{array}{l}\text { Total de itens disponíveis de } \\
\text { infraestrutura }\end{array}$ \\
\hline
\end{tabular}

Continua 


\begin{tabular}{|lll|}
\hline $\begin{array}{l}\text { Variável } \\
\text { Independente }\end{array}$ & Parâmetros para definição dos indicadores & Indicador \\
\hline $\begin{array}{l}\text { Tempo de } \\
\text { Permanência }\end{array}$ & - Tempo de permanência do médico na equipe & $\begin{array}{l}\text { Tempo de permanência do } \\
\text { médico na equipe de saúde } \\
\text { em meses }\end{array}$ \\
\hline Formação & - Título de especialista em medicina da família e comunidade & $\begin{array}{l}\text { Total de médicos especialistas } \\
\text { em medicina da família e } \\
\end{array}$ \\
& & comunidade \\
\hline
\end{tabular}

Fonte: Elaborada pelos autores.

\subsection{Análise dos dados}

A análise dos dados foi realizada em duas etapas. Na primeira etapa, foi mensurado o desempenho dos médicos da ESF considerando o cumprimento de suas atribuições nos princípios ordenadores. Foi considerado desempenho esperado o cumprimento de todas as atribuições do médico da SF. Assim, foram comparados o desempenho esperado e o desempenho realizado. O desempenho foi considerado efetivo nos casos em que houve o cumprimento integral das atribuições do médico. Para isso, foi utilizado um indicador que indicava o total de cumprimento de ações diante do total esperado.

Com isso, foi possível verificar a contribuição do médico para a consecução dos princípios ordenadores e identificar lacunas de desempenho. Conforme aponta Rocha (2011), o cumprimento dos princípios ordenadores é fundamental para se pensar em resultados na AP. Segundo o autor, o cumprimento eficiente do processo de trabalho inerente aos princípios ordenadores contribui para a atuação eficaz dos profissionais que compõem a equipe de saúde e, consequentemente, interfere nos indicadores de saúde populacional.

Os resultados do processo de mensuração do desempenho do médico da SF foram apresentados por distrito sanitário. Segundo Barbosa e colaboradores (2011), a avaliação comparativa do desempenho representa um instrumento interessante do ponto de vista da gestão, na medida em que proporciona informações sobre as regiões e unidades que apresentam melhor desempenho, podendo servir, inclusive, de referência.

Após a aferição do desempenho do profissional médico, foi realizada a segunda etapa da pesquisa com o intuito de verificar se as variáveis, desempenho da equipe de Saúde da Família, formação profissional, tempo de permanência do profissional na equipe de SF e infraestrutura da Unidade Básica de Saúde interferem no desempenho do médico da Estratégia Saúde da Família. As etapas de análise dos dados podem ser observadas, conforme figura 1.

Observa-se na figura 1 que a análise do desempenho do médico da saúde foi realizada considerando os indicadores de desempenho inerentes aos princípios ordenadores. Na segunda etapa, buscou-se analisar se a variável dependente, desempenho do médico, é 
influenciada pelas variáveis independentes. Somente após a análise da relação de dependência é que se pode afirmar se as variáveis preditoras em questão interferem ou não no desempenho do médico. A análise de regressão múltipla foi utilizada para investigar se há ou não essa relação de dependência.

\section{Figura 1}

Etapas de análise

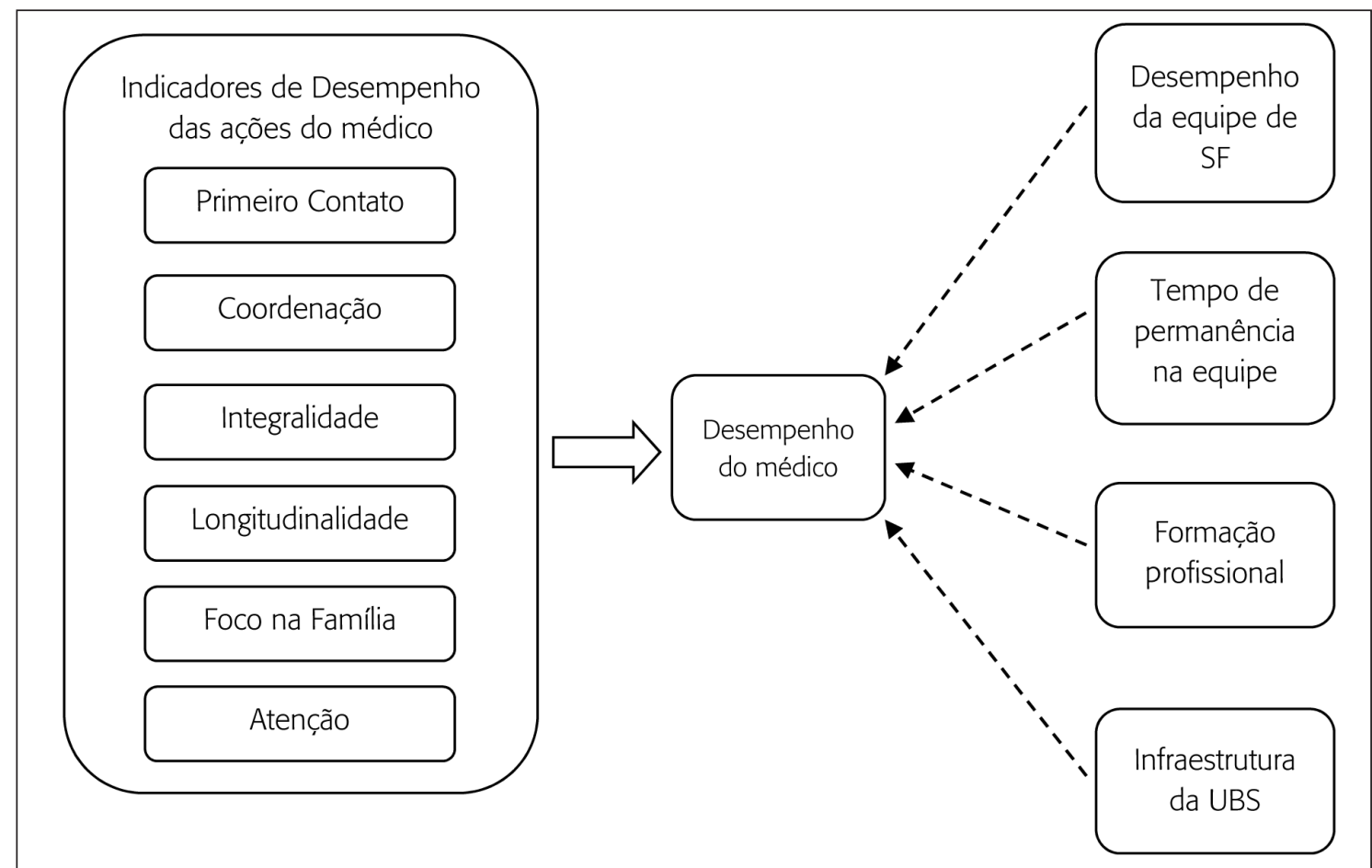

Fonte: Elaborada pelos autores.

Entre as 512 equipes analisadas, foram identificados, inicialmente, 483 casos de equipes que contavam com médico em sua composição. Dessas equipes, somente 352 apresentaram todos os dados referentes aos indicadores das variáveis analisadas. Optou-se por retirar todos os missings da base de dados para não comprometer o processo de modelagem e para preservar os dados a fim de refletir a realidade da forma mais próxima possível. A tabela 1 mostra a distribuição dos médicos por distrito sanitário de Belo Horizonte, segundo amostra desta pesquisa. 
Tabela 1

Número de médicos da saúde da família, por distrito sanitário de Belo Horizonte

\begin{tabular}{|lcr|}
\hline Distrito & Médicos & $\%$ \\
\hline Barreiro & 46 & 13,07 \\
Centro-Sul & 21 & 5,97 \\
Leste & 27 & 7,67 \\
Nordeste & 54 & 15,34 \\
Noroeste & 56 & 15,91 \\
Norte & 34 & 9,66 \\
Oeste & 47 & 13,35 \\
Pampulha & 16 & 4,55 \\
Venda Nova & 51 & 14,49 \\
Total & 352 & 100,00 \\
\hline
\end{tabular}

Fonte: Elaborada pelos autores a partir da base de dados da pesquisa "Monitoramento de resultados e desempenho e satisfação dos usuários da estratégia saúde da família: um estudo em Belo Horizonte".

\section{Apresentação e análise dos resultados}

Primeiramente, apresenta-se o resultado do desempenho dos médicos, considerando o cumprimento de suas práticas e atribuições. A descrição da performance dos médicos da SF foi realizada por distrito sanitário de Belo Horizonte, a fim de possibilitar a comparação da performance dos médicos em cada distrito. Essa comparação permite identificar os distritos nos quais há maior deficiência de desempenho do médico e aqueles em que há os melhores índices de performance do médico. Após aferir o desempenho do médico, procedeu-se à análise de regressão múltipla, a fim de verificar se as variáveis se correlacionavam com o desempenho do médico no trabalho.

\subsection{Desempenho do médico da saúde da família}

Para mensurar a performance dos médicos da saúde da família foi considerado o cumprimento dos 63 indicadores analisados, que incluem as atribuições e os procedimentos dos médicos com base nos princípios ordenadores. Observa-se percentual médio de cumprimento superior a $80 \%$ nos distritos sanitários. Esses resultados podem ser observados na tabela 2. 
Em média, os médicos das equipes avaliadas desempenhavam em sua rotina de atendimento 54,03 atividades de processo de trabalho, entre as 63 possíveis. Essa proporção corresponde a um cumprimento médio de $85,76 \%$ das atividades que devem ser desempenhadas por essa categoria profissional. Observa-se também que a média de desempenho dos médicos da ESF por distrito sanitário varia pouco, de 51,50 a 55,94. O mesmo foi observado por Barbosa e colaboradores (2011) em relação ao desempenho das equipes. Os autores sugerem que essa hegemonia pode ser em parte explicada pela influência das políticas de saúde do município de Belo Horizonte.

Tabela 2

Desempenho do médico da saúde da família, por distrito sanitário

\begin{tabular}{|lccccc|}
\hline & \multicolumn{5}{c}{ Desempenho } \\
\cline { 2 - 6 } & $\begin{array}{c}\text { Cumprimento \% dos indicadores } \\
\text { de processo de trabalho }\end{array}$ & $\begin{array}{c}\text { Média de indicadores } \\
\text { cumpridos }\end{array}$ & $\begin{array}{c}\text { Desvio- } \\
\text { padrão }\end{array}$ & Mínimo & Máximo \\
\hline Barreiro & $81,75 \%$ & 51,50 & 2,88 & 47 & 59 \\
Centro-Sul & $84,13 \%$ & 53,00 & 3,73 & 47 & 60 \\
Leste & $84,66 \%$ & 53,33 & 3,77 & 48 & 63 \\
Nordeste & $88,80 \%$ & 55,94 & 3,32 & 48 & 62 \\
Noroeste & $84,44 \%$ & 53,20 & 3,05 & 47 & 59 \\
Norte & $86,04 \%$ & 54,21 & 3,13 & 48 & 60 \\
Oeste & $85,55 \%$ & 53,89 & 3,57 & 47 & 62 \\
Pampulha & $86,51 \%$ & 54,50 & 2,83 & 50 & 59 \\
Venda Nova & $88,67 \%$ & 55,86 & 3,11 & 47 & 62 \\
Belo Horizonte & $85,76 \%$ & 54,03 & 3,53 & 47 & 63 \\
\hline
\end{tabular}

Fonte: Dados da Pesquisa.

Os resultados encontrados demonstraram que os médicos apresentam desempenho satisfatório ao considerar suas práticas com base nos princípios norteadores do modelo da ESF. Conforme pontuam Barbosa e colaboradores (2011), esses resultados demonstram que o modelo de ESF no município caminha para um cenário de consolidação de práticas embasadas em processo de trabalho diferenciado. Os resultados apresentados são um indicativo de que os profissionais médicos contribuem para o cumprimento dos princípios que regem o funcionamento da ESF.

Conforme destacam Milkovich e Boudreau (2010), é importante analisar os resultados obtidos no processo de mensuração de desempenho para verificar quais fatores podem influenciar o desempenho dos profissionais, buscando, assim, corrigir possíveis lacunas de desempenho e fornecer bases para a tomada de decisão.

A partir dessa perspectiva, e considerando alguns fatores listados pela literatura de saúde, buscou-se averiguar como a performance do médico é influenciada por variáveis independentes escolhidas. Para isso foi realizada a análise de regressão linear múltipla. 


\subsection{Resultados da análise de regressão múltipla}

Hair e colaboradores (2009) pontuam que a regressão também avalia o grau e o caráter da relação entre as variáveis dependentes e independentes por meio do exame da magnitude, do sinal e da significância estatística do coeficiente de regressão de cada variável independente. Os autores salientam que, para fins de explicação, os coeficientes de regressão se tornam indicadores do impacto relativo e da importância das variáveis independentes em sua relação com a variável dependente.

De acordo com Hair e colaboradores (2009), a análise de regressão múltipla é uma técnica estatística que pode ser utilizada para mensurar a relação entre uma única variável dependente métrica e duas ou mais variáveis independentes. Revela-se, portanto, adequada para este estudo, já que se busca interpretar como as variáveis independentes explicam a variação da variável dependente desempenho do médico da SF. Para isso, procedeu-se à análise do banco de dados, bem como ao descritivo das variáveis envolvidas no processo de modelagem. Cada uma dessas etapas pode ser observada a seguir.

Para mensurar o desempenho do médico da SF, foram utilizados os indicadores de atividades e processos de trabalho, considerando as atribuições desses profissionais em relação aos princípios ordenadores, apontados no quadro 2. Para mensurar o desempenho da equipe de $\mathrm{SF}$, foram utilizados 155 indicadores das atribuições e do processo de trabalho dos membros da equipe, apontados no quadro $3 .{ }^{1}$ Quanto mais próximo de 155 , melhor é o desempenho da equipe. A infraestrutura foi mensurada considerando 170 itens referentes à estrutura física da UBS, entre insumos, materiais e equipamentos. A escala utilizada foi de 0 a 170 . Quanto mais próximo de 170 , melhores as condições de trabalho. O tempo de permanência na equipe de Saúde da Família foi mensurado considerando o período, em meses, em que o médico integra a equipe. Por meio da variável formação do médico, buscou-se identificar os médicos que possuem formação generalista. Para isso foi considerado o indicador: título de especialista em medicina da família e comunidade.

Na tabela 3 apresentam-se os dados descritivos das variáveis quantitativas estudadas: desempenho do médico da ESF, desempenho da equipe de SF, infraestrutura e tempo de permanência na equipe de SF.

Em média, os médicos da estratégia saúde da família cumprem 54,05 atribuições, o que equivale a $85,79 \%$ do total de 63 atribuições e procedimentos observados. Isso demonstra um bom desempenho dos médicos da SF de Belo Horizonte. O cumprimento mínimo observado é 47 (74,60\%) e o máximo é 63 (100\%). Em relação ao desempenho da equipe, nota-se que, em média, 76,75\% do total de 155 atribuições e procedimentos analisados foram cumpridos. Isso corresponde ao cumprimento de 118,97 atribuições e procedimentos da equipe de saúde. O cumprimento máximo observado das atribuições da equipe é de 95,48\%, ou seja, 148 atribuições. Já o cumprimento mínimo observado é 87 (56,13\%).

\footnotetext{
${ }^{1}$ Enfermeiro, auxiliar de enfermagem e agentes comunitários de saúde.
} 
Tabela 3

Descritivo das variáveis quantitativas

\begin{tabular}{|lcccc|}
\hline $\begin{array}{l}\text { Características } \\
\text { Estatísticas }\end{array}$ & $\begin{array}{c}\text { Desempenho do } \\
\text { Médico da SF }\end{array}$ & $\begin{array}{c}\text { Desempenho da } \\
\text { Equipe de SF }\end{array}$ & $\begin{array}{c}\text { Tempo de } \\
\text { Infraestrutura }\end{array}$ & $\begin{array}{c}\text { Permanência na } \\
\text { Equipe (meses) }\end{array}$ \\
\hline N & 352 & 352 & 352 & 352 \\
Média & 54,05 & 118,97 & 126,7 & 36,48 \\
Mediana & 54 & 118 & 127 & 30 \\
Desvio-padrão & 3,55 & 12,12 & 8,4 & 32,18 \\
Variância & 12,59 & 146,88 & 70,48 & 1035,6 \\
Mínimo & 47 & 87 & 112 & 1 \\
Máximo & 63 & 148 & 149 & 104 \\
1o Quartil & 51 & 111 & 121 & 8 \\
2o Quartil & 54 & 118 & 127 & 30 \\
3o Quartil & 57 & 126,75 & 133 & 60 \\
\hline
\end{tabular}

Fonte: Dados da pesquisa.

As condições de trabalho observadas foram bem avaliadas, apresentando média de $74,53 \%(126,7)$ de adequação da estrutura física, considerando os 170 itens analisados. Observa-se também que o tempo médio de permanência do médico na equipe é de 36,48 meses. O tempo máximo de permanência observado é de 104 meses e o mínimo é de 1 mês.

A formação do médico é a única variável não métrica do modelo. Ela objetiva identificar se o médico da saúde da família possui formação generalista e averiguar se essa formação influencia sua performance na SF. Segundo Starfield (2002), a formação generalista é mais indicada para os médicos que atuam na Atenção Básica, já que eles lidam com uma variedade maior de doenças. Para mensurar essa variável foi utilizado o indicador: título de especialista em medicina da família e comunidade. A partir da frequência de distribuição dessa variável observou-se que $52,56 \%$ dos médicos que atuam na SF não possuem título de especialista em medicina da família e comunidade, já 47,44\% possuem esse título.

Antes de iniciar o processo de modelagem buscou-se verificar se a variável dependente, desempenho do médico da ESF, atendia ao pressuposto de normalidade, tendo em vista que o modelo de regressão só se aplica quando há normalidade na distribuição dos dados da variável dependente.

Para analisar a distribuição dos dados da variável dependente, desempenho do médico, optou-se por utilizar os gráficos histograma e boxplot. Observa-se pelo boxplot que 50\% dos 
dados intermediários da distribuição concentram-se entre o primeiro quartil e o terceiro quartil (Anderson et al., 2011). A figura 2 permite inferir que a distribuição dos dados representados pelo histograma sugere uma distribuição normal. Isso também é sugerido ao se observar a distribuição dos dados pelo boxplot. Há indícios de simetria dos dados compreendidos entre o primeiro quartil e a mediana e entre a mediana e o terceiro quartil.

Figura 2

Distribuição dos dados do desempenho do médico da saúde da família

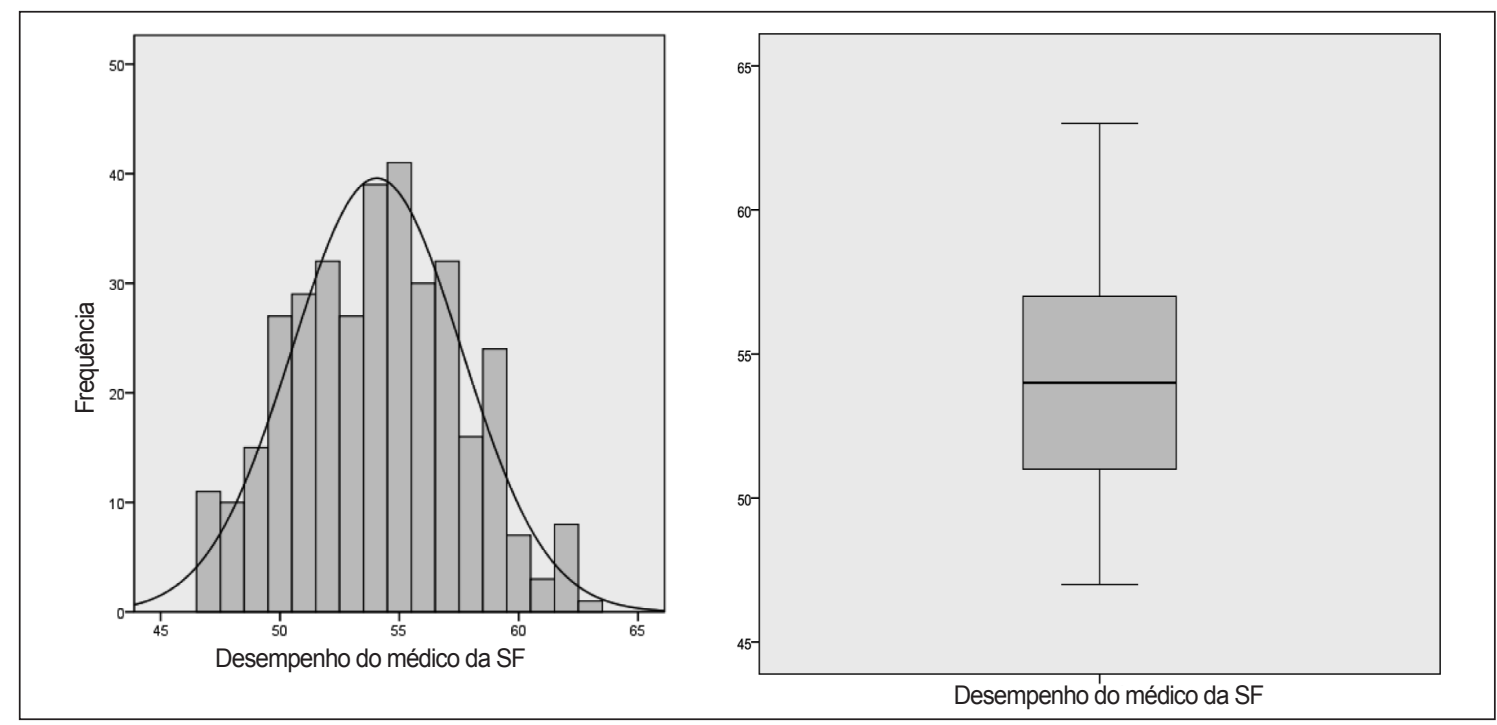

Fonte: Dados da pesquisa.

Mesmo observando indícios de normalidade na distribuição dos dados, fez-se necessário confirmar essa análise. Para isso, aplicou-se o teste de normalidade Kolmogorov-Smirnov. O coeficiente de confiança utilizado no teste foi de $95 \%$. Esse coeficiente de confiança foi adotado em todos os testes estatísticos aplicados neste estudo. O resultado desse teste de normalidade comprova que a variável — desempenho do Médico da ESF — atende ao pressuposto de normalidade, já que o p-valor encontrado, de 0,058 , indica que os dados se originam de uma distribuição normal.

Ainda na fase de pré-modelagem, procurou-se verificar se as variáveis independentes são correlacionadas. Segundo Hair e colaboradores (2009), a multicolinearidade ocorre quando qualquer variável independente é altamente correlacionada com um conjunto de outras variáveis independentes, e isso reduz o poder preditivo/explicativo de qualquer variável independente, na medida em que ela é associada a outras variáveis independentes. Além disso, pode ter efeitos consideráveis sobre a estimação dos coeficientes de regressão e seus testes de significância estatística (Hair et al., 2009). 
Para avaliar a existência de correlação entre as variáveis, utilizou-se o teste de correlação de Pearson (tabela 4). Ao analisar o nível de significância entre as variáveis independentes, observou-se que a variável desempenho da equipe de SF apresenta correlação com a variável infraestrutura, uma vez que apresenta p-valor igual a 0,000, inferior, portanto, a 5\%. Por meio do coeficiente de correlação de Pearson $(-0,285)$, nota-se que a correlação entre essas variáveis independentes é baixa e inversamente proporcional.

Tabela 4

Análise de correlação entre as variáveis independentes

\begin{tabular}{|c|c|c|c|c|}
\hline Variável Independente & Teste & $\begin{array}{l}\text { Desempenho da } \\
\text { Equipe de SF }\end{array}$ & Infraestrutura & $\begin{array}{c}\text { Tempo de Permanência } \\
\text { na Equipe }\end{array}$ \\
\hline \multirow{2}{*}{$\begin{array}{l}\text { Desempenho da Equipe } \\
\text { de SF }\end{array}$} & Correlação de Pearson & 1 & $-0,285^{*}$ & $-0,078$ \\
\hline & p-valor & & 0,00 & 0,146 \\
\hline \multirow{2}{*}{ Infraestrutura } & Correlação de Pearson & $-0,285^{*}$ & 1 & 0,075 \\
\hline & p-valor & 0,000 & & 0,161 \\
\hline \multirow{2}{*}{$\begin{array}{l}\text { Tempo de Permanência na } \\
\text { Equipe }\end{array}$} & Correlação de Pearson & $-0,078$ & 0,075 & 1 \\
\hline & p-valor & 0,146 & 0,161 & \\
\hline
\end{tabular}

Fonte: Dados da pesquisa.

Utilizou-se, inicialmente, a análise de regressão simples, que consiste na aplicação do modelo de regressão da variável dependente, desempenho do médico da ESF, a cada variável independente. Adotou-se esse procedimento para verificar se cada variável preditora ajuda a explicar individualmente a variação da variável dependente e, também, para selecionar quais variáveis independentes irão compor o modelo múltiplo. Foram consideradas importantes significativamente as variáveis que apresentaram nível de significância inferior a 0,20 .

A partir dos resultados obtidos na análise de regressão univariada (tabela 5), verificouse que as variáveis independentes desempenho da equipe de SF, infraestrutura e tempo de permanência na equipe de SF foram consideradas significativas. Portanto, entram na composição do modelo múltiplo, que busca identificar as variáveis que explicam a variação no desempenho do médico de SF. Já a variável formação do médico não se mostrou significativa estatisticamente, considerando os indicadores e a amostra utilizados neste estudo. Cabe ressaltar que a variável formação utilizada para identificar os médicos que possuem formação generalista levou em consideração apenas os médicos que possuem título de "especialista em medicina da família e comunidade"; não foi avaliado se os médicos possuíam formação generalista na especialidade clínica médica, por exemplo. Isso pode ter interferido no total de médicos da amostra que possuem formação generalista e também influenciado no resultado da análise de regressão. 
Tabela 5

Análise univariada

\begin{tabular}{|lcc|}
\hline Variável Independente & Beta & p-valor \\
\hline Desempenho da Equipe de SF & 0,532 & 0,000 \\
Infraestrutura & $-0,175$ & 0,001 \\
Tempo de Permanência na Equipe & $-0,094$ & 0,079 \\
$\begin{array}{l}\text { Título de Especialista em Medicina da Família } \\
\text { e Comunidade }\end{array}$ & $-0,037$ & 0,49 \\
\hline
\end{tabular}

Fonte: Dados da pesquisa.

Após identificar quais variáveis independentes ajudaram a explicar a variação do desempenho do médico individualmente, aplicou-se o modelo de regressão múltipla. Nesse caso, entraram na análise de regressão as variáveis independentes: desempenho da equipe de SF, infraestrutura da UBS e tempo de permanência na equipe de SF.

Segundo Hair e colaboradores (2009), quatro suposições devem ser examinadas: linearidade do fenômeno medido; homocedasticidade; independência dos termos de erro; e normalidade da distribuição dos termos de erro. Segundo o autor, o método de diagnóstico de resíduos é um método básico para verificar violações de suposições para a relação geral. Assim, a análise gráfica de resíduos foi utilizada para verificar se as suposições do modelo de regressão múltipla foram atendidas. Além dos pressupostos analisados, a alta correlação entre as variáveis independentes também pode afetar a estimação e a interpretação dos resultados da regressão múltipla. Para verificar a existência de multicolinearidade, foi observado o Fator de Inflação da Variância (VIF).

De acordo com a tabela 6, o valor do VIF encontrado foi de 1,092 para as variáveis desempenho da equipe de SF e infraestrutura, e de 1,009 para a variável tempo de permanência na equipe de saúde da família. Como esses valores se aproximam de 1,0, indicam baixa multicolinearidade. Nota-se, assim, que a correlação entre as variáveis independentes não interfere nos resultados da regressão.

Tabela 6

Análise de multicolinearidade

\begin{tabular}{|lc|}
\hline Variáveis Independentes & VIF \\
\hline Desempenho da Equipe de SF & 1,092 \\
Infraestrutura & 1,092 \\
Tempo de Permanência na Equipe de SF & 1,009 \\
\hline
\end{tabular}

Fonte: Dados da pesquisa. 
Após testar todos os pressupostos do modelo de regressão e de constatar que eles foram atendidos, é possível certificar-se da precisão dos resultados da análise de regressão múltipla e, assim, interpretar os resultados obtidos.

Os resultados da análise de regressão múltipla indicaram que o desempenho da equipe de Saúde da Família foi a única variável que explicou, estatisticamente, a variação do desempenho do médico da SF. Isso pode ser observado pela tabela 7 , em que o p-valor do desempenho da equipe de SF é de 0,000. Além disso, observa-se que a variação de uma unidade no desempenho do médico da ESF é associada à variação de 0,521 do desempenho da equipe.

Tabela 7

Análise multivariada

\begin{tabular}{|lcc|}
\hline Variáveis Independentes & Beta & p-valor \\
\hline Desempenho da Equipe de SF & 0,521 & 0,000 \\
Infraestrutura & $-0,023$ & 0,627 \\
Tempo de Permanência na Equipe & $-0,052$ & 0,257 \\
\hline
\end{tabular}

Fonte: Dados da pesquisa.

As demais variáveis - infraestrutura e tempo de permanência do médico na equipe de SF — não foram consideradas estatisticamente significantes, tendo em conta a amostra e os indicadores utilizados neste estudo. O coeficiente de determinação ajustado encontrado nesta modelagem foi de 0,28 , o que significa que $28 \%$ da variação do desempenho do médico ESF pode ser explicada pela variação do desempenho da equipe de saúde da família. Em ciências sociais, esse percentual é bastante significativo. Além disso, é preciso destacar que o desempenho dos indivíduos no trabalho sofre influência de outros fatores, o que torna ainda mais significativo o resultado apresentado.

Tabela 8

Coeficiente de determinação

\begin{tabular}{|cc|}
\hline Coeficiente de determinação & Coeficiente de determinação ajustado \\
\hline 0,286 & 0,280 \\
\hline
\end{tabular}

Fonte: Dados da Pesquisa.

A partir da análise de regressão múltipla, observou-se que, das variáveis independentes analisadas neste estudo, apenas o desempenho da equipe de saúde da família, por ser significante estatisticamente, influenciou o desempenho do médico da ESF. Ou seja, a variação do desempenho do médico pode ser explicada em parte pela variação da performance da equipe de SF. Os resultados apresentados neste estudo demonstraram também 
que as demais variáveis independentes - adequação da estrutura física, disponibilidade de insumos, materiais e equipamentos, formação do médico da família e tempo de permanência deste profissional na equipe de SF — não contribuíram para explicar a variação da performance do médico.

\section{Considerações finais}

A SF tem se apoiado em um conjunto de princípios ordenadores que regem seu funcionamento e forma de organização. Conforme observa Starfield (2002), o cumprimento dos princípios ordenadores vem sendo utilizado como balizador da efetividade e da qualidade dos serviços na Atenção Primária à Saúde. A partir dos objetivos da ESF, observa-se a relevância de mensurar o desempenho dos profissionais de saúde, principalmente o médico, para a consecução de seus resultados. A AP, por ser um serviço intensivo em mão de obra (Mendes, 2002) e por gerar produtos não materiais, depende das habilidades e dos conhecimentos de seus profissionais para alcançar os resultados esperados.

Apesar da relevância do tema estudado, ainda há poucos estudos direcionados para análise da contribuição dos profissionais de saúde para os resultados da Estratégia Saúde da Família. O exame de como aspectos vinculados à GRH se relacionam com aspectos de resultado em saúde ainda é um campo incipiente que carece de evidências. Ao se debruçar sobre a temática de desempenho dos profissionais e sua respectiva associação com os resultados do processo de oferta de serviços, as evidências são ainda mais escassas.

Os estudos que têm sido realizados, para avaliar as contribuições fornecidas pelas práticas GRH para com os resultados em saúde, com base em uma literatura recente, têm produzido resultados por vezes contraditórios. Alguns encontram associações entre a densidade de profissionais e resultados em saúde (Anand e Bärnighausen, 2004) e (Chen et al., 2004), outros relatam o contrário, como os estudos de Robinson e Wharrad (2000, 2001), que não encontraram associação entre os profissionais de enfermagem e os indicadores de mortalidade infantil. A respeito do desempenho do médico, a maior parte dos estudos da literatura internacional busca abordar os impactos de programas de remuneração por desempenho, sem tratar sobre quais elementos contribuem para explicar um bom ou mau resultado das ações desenvolvidas por esse profissional. No Brasil não foram observados estudos sobre o desempenho dos médicos, considerando a contribuição desses profissionais para o alcance dos resultados almejados pela ESF.

Há carência de processos avaliativos que buscam vincular o desempenho dos profissionais aos resultados do sistema de saúde. Considerando a importância do médico da família para o adequado funcionamento da ESF, este estudo averiguou a contribuição da performance dos médicos das equipes de saúde de Belo Horizonte para o cumprimento dos princípios ordenadores. A influência do desempenho da equipe, observada neste estudo, reforça, mais uma vez, a importância dessa nova forma de organização do trabalho no sistema de saúde, 
tendo em vista que o trabalho em equipe é um dos pilares de sustentação da ESF. Isso permite inferir que esse modelo de atenção à saúde, baseado na organização do trabalho em equipe multiprofissional, tendo como foco a organização do trabalho e a prestação de serviços à família e à comunidade em que os usuários vivem, tende a apresentar bons resultados em termos de serviços de saúde efetivo e de qualidade para a população usuária. Esse achado inicial permite suscitar o debate sobre como o bom desempenho do médico, articulado com uma atuação satisfatória dos demais membros das equipes de saúde, pode se relacionar com os resultados epidemiológicos de saúde populacional.

Os resultados obtidos no processo de mensuração permitem a identificação de gaps de desempenho, fornecendo bases para a tomada de decisão, mas para isso é preciso analisar quais fatores podem ter influenciado a performance dos profissionais no trabalho. Nessa perspectiva, além de mensurar o desempenho do médico, buscou-se verificar se o desempenho desse profissional é influenciado por algumas variáveis discutidas na literatura da saúde.

A análise de regressão múltipla demonstrou que apenas o desempenho da equipe de SF influencia a performance do médico no trabalho. Esse resultado reforça a importância dessa nova forma de organização do trabalho, baseada em uma equipe multiprofissional, na qual todos os profissionais da equipe atuam de forma integrada, viabilizando a identificação de estratégias para a promoção de melhorias a fim de oferecer serviço de qualidade e de forma integral para a população usuária. Isso demonstra que os demais profissionais da equipe são essenciais não apenas para o alcance dos resultados dos serviços de saúde, mas também para potencializar o desempenho dos médicos da SF. Vale ressaltar que esses resultados se referem à amostra utilizada neste trabalho e, também, aos indicadores utilizados para mensurar tanto a variável dependente (desempenho do médico da ESF) quanto as variáveis preditoras.

Entre as limitações do presente estudo, destacam-se as restrições quanto à potencialidade de generalização dos resultados encontrados, tendo em vista que os resultados alcançados neste estudo foram contextualizados para Belo Horizonte e seu entendimento em outros contextos pode ser diferenciado.

Outro ponto que vem ganhando destaque se refere à possibilidade de realização de análises considerando uma perspectiva multinível (Ostroff e Bowen, 2000), na qual os resultados são escalonados em níveis antes da concepção de um modelo avaliativo global do desempenho do profissional médico, por exemplo. Para tanto, têm sido utilizadas técnicas como a análise de equações estruturais, análise multinível, que não puderam ser empregadas no presente trabalho. Cabe destacar ainda como desafio futuro a importância de se incorporarem resultados de saúde populacional que ajudem a entender como o desempenho do médico modula a obtenção de resultados junto a indicadores de saúde populacional.

Por fim, espera-se que este estudo possa contribuir para suscitar a discussão sobre o desempenho médico e seus reflexos sobre os resultados dos serviços de saúde da ESF, tendo em vista o protagonismo desse profissional na prestação de serviços de saúde. Além de incentivar 
novas publicações sobre o tema utilizando outras metodologias; e abrir perspectivas de reflexões sobre novas formas de inserção do profissional médico, considerando, por exemplo, a entrada de novos atores no processo, decorrente de ações governamentais específicas voltadas para suprir as dificuldades de prestação de serviços.

\section{Referências}

ABBAD, Gardênia S.; FREITAS, Isa A.; PILATI, Ronaldo. Contexto de trabalho, desempenho competente e necessidades em TD\&E. In: BORGES-ANDRADE, Jairo E.; ABBAD, Gardênia S.; MOURÃO, Luciana. Treinamento, desenvolvimento e educação em organizações e trabalho. Porto Alegre: Artmed, 2006. cap 12, p. 231-254.

ANAND, Sudir; BÄRNIGHAUSEN, Till. Human resources and health outcomes: cross-country econometric study. Lancet, v. 364, n. 9445, p. 1603-1609, 2004.

ANDERSON, David R.; SWEENEY, Dennis J.; WILLIAMS, Thomas A. Estatística aplicada à administração e economia. 2. ed. São Paulo: Cengage Learning, 2011.

BARBOSA, Allan C. Q. et al. Monitoramento de resultados, desempenho e satisfação dos usuários da estratégia Saúde da Família. Pensar BH. Política Social, v. 28, p. 42-45, 2011.

BRANDÃO, Hugo P.; GUIMARÃES, Tomás A. Gestão de competências e gestão de desempenho: tecnologias distintas ou instrumentos de um mesmo construto? Rev. Adm. Empres., v. 41, n. 1, p. 8-15. 2001.

BRASIL. Lei no 8.080 de 19 de setembro de 1990. Diário Oficial da República Federativa do Brasil, Brasília, 1990.

BRASIL. Ministério da Saúde. Secretaria de Atenção à Saúde. Departamento de Atenção Básica. Política nacional de atenção básica. Brasília: Ministério da Saúde, 2012.

BRASIL. Saúde da Família: uma estratégia para a reorientação do modelo assistencial. Brasília: Ministério da Saúde. 1997.

BUCHAN, James; FRONTEIRA, Ines; DUSSAULT, Gilles. Continuity and change in human resources policies for health: lessons from Brazil. Hum Resour Health, v. 9, p. 17, 2011.

CAPOZZOLO, Angela A. No olho do furacão: trabalho médico e o programa de Saúde da Família. Tese (doutorado em saúde coletiva) — Faculdade de Ciências Médicas, Universidade Estadual de Campinas, Campinas, 2003.

CASSEL, Christine K.; JAIN, Sachin H. Assessing individual physician performance does measurement suppress motivation? JAMA, v. 307, n. 24, p. 2595-1596, 2012.

CHEN, Lincoln et al. Human resources for health: overcoming the crisis. Lancet, v. 364 n. 27, p. 1984-1990, 2004. 
DE VRIES, Han et al. International comparison of ten medical regulatory systems: Egypt, Germany, Greece, India, Italy, Nigeria, Pakistan, Poland, South Africa and Spain. Santa Monica, California: Rand Corporation; 2009.

EXWORTHY, Mark et al. The role of performance indicators in changing the autonomy of the general practice profession in the UK. Social Science \& Medicine, v. 56, p. 1493-1504, 2003.

GIL, Antônio C. Métodos e técnicas de pesquisa social. São Paulo. Atlas, 2006.

GOLDENBERG, Mirian. A arte de pesquisar: como fazer pesquisa qualitativa em ciências sociais. 6 . ed. Rio de Janeiro: Record, 2002.

GONÇALVES, Carlos A.; MEIRELLES, Anthero M. Projetos e relatórios de pesquisa em administração. São Paulo: Atlas, 2004.

GRACE, Elizabeth S.; WENGHOFER, Elizabeth F.; KORINEK, Elizabeth J. Predictors of physician performance on competence assessment: findings from CPEP, the Center for Personalized Education for Physicians. Academic Medicine, v. 89, n. 6, p. 912-9, 2014.

GROL, Richard. Changing physicians' competence and performance: finding the balance between the individual and the organization. Journal of Continuing Education in the Health Professions, v. 22, p. 244-251, 2002.

GYORGYI, Albert S. Necessidade de pesquisa em atenção primária. In: STARFIELD, Bárbara. Atenção primária: equilíbrio entre necessidades de saúde, serviços e tecnologia. Brasília: Unesco; Ministério da Saúde, 2002.

HAIR, Joseph F. et al. Análise multivariada de dados. 6. ed. Porto Alegre: Bookman, 2009.

HOGG, William et al. Framework for primary care organizations: the importance of a structural domain international. Journal for Quality in Health Care, v. 20, n. 5, p. 308-313, 2008.

MAGALHÃES JÚNIOR, Helvécio M. Desafios e inovações na gestão do SUS em Belo Horizonte: a experiência de 2003 a 2008. Belo Horizonte: Mazza, 2010.

MARSHALL, Susan; HAYWOOD, Kirstie; FITZPATRICK, Ray. Impact of patient-reported outcome measures on routine practice: a structured review. Journal of Evaluation of Clinical Practice, v. 12, n. 5, p. 559-568, 2006.

MENDES, Eugênio V. A atenção primária à saúde no SUS. Fortaleza: Escola de Saúde Pública do Ceará, 2002.

MILKOVICH, George T.; BOUDREAU, John W. Administração de recursos humanos. São Paulo: Atlas, 2010.

OLIVEIRA-CASTRO, Gardênia A.; LIMA, Geny B. C.; VEIGA, Maria R. M. Implantação de um sistema de avaliação de desempenho: métodos e estratégias. Revista de Administração da Universidade de São Paulo, v. 31, n. 3, p. 38-52, 1996. 
OSTROFF, Cheri; BOWEN, David. Moving HR to a higher level: HR practices and organizational effectiveness. In: KLEIN, Katherine; KOZLOWSKI, Steve (Ed.). Multilevel theory, research, and methods in organizations. San Francisco, CA: Jossey Bass, 2000. 211-266.

PUSTAI, Oldaci J. O sistema de saúde no Brasil. In: DUNCAN, Bruce B.; SCHMIDT, Maria I.; GIUGLIANI, Elsa R. J. (Org.). Medicina ambulatorial: condutas de atenção primária baseada em evidências. 3. ed. Porto Alegre: Artmed, 2004. 71-82.

ROBINSON, Jane; WHARRAD, Heather. Invisible nursing: exploring health outcomes at a global level-relationships between infant and under-5 mortality rates and the distribution of health professionals, GNP per capita, and female literacy. JAN, v. 32, p. 28-40, 2000.

ROBINSON, Jane; WHARRAD, Heather. The relationship between attendance at birth and maternal mortality rates: an exploration of United Nations' data sets including the ratios of physicians and nurses to population, GNP per capita and female literacy. JAN, v. 31, p. 445-55, 2001.

ROCHA, Thiago A. H. Gestão de recursos humanos e estratégia Saúde da Família - um estudo em Belo Horizonte pela ótica da Avaliação de Eficiência. Dissertação (mestrado em administração) Faculdade de Ciências Econômicas, Universidade Federal de Minas Gerais, Belo Horizonte, 2011.

ROCHA, Thiago A. H. et al. Human resource management in health and performance of work process in the primary health care-an efficiency analysis in a Brazilian municipality. Journal of Health Management, v. 16, p. 365-379, 2014.

RODRIGUES, Raquel B. et al. Formação de médicos especialistas: lições para os sistemas de saúde. In: BARBOSA, Allan C. Q.; SILVA, Joaquim R. (Org.). Economia, gestão e saúde: as relações luso-brasileiras em perspectiva. Lisboa: Colibri, 2011. p. 215-238.

ROESCH, Sylvia M. A. Projetos de estágio e de pesquisa em administração. 3. ed. São Paulo: Atlas, 2010.

RUSSELL, Grant M. et al. Managing chronic disease in Ontario primary care: the impact of organizational factors. Annals Family Medicine, v. 7, n. 4, p. 309-318, 2009.

SELLTIZ, Claire et al. Métodos de pesquisa das relações sociais. São Paulo: Herder, 1965.

SILVA, Núbia C. Gestão de recursos humanos na saúde da família: fato ou ficção? Belo Horizonte: Observatório de Recursos Humanos em Saúde do Nig.One/UFMG, 2009.

SONNENTAG, Sabine; FRESE, Michael. Performance concepts and performance theory. In: SONNENTAG, Sabine (Org.). Psychological management of individual performance. Chichester, West Sussex, UK: 2002. p. 3-25.

STARFIELD, Bárbara. Atenção primária: equilíbrio entre necessidades de saúde, serviços e tecnologia. Brasília: Unesco; Ministério da Saúde, 2002.

TEIXEIRA, Marcelo G. Construindo a política pública de saúde: o SUS no município em Belo Horizonte. Política Social, v. 28, p. 5-9, 2011. 
WRIGHT, Christine et al. Multisource feedback in evaluating the performance of doctors: the example of the UK general medical council patient and colleague questionnaires. Academic Medicine, v. 87, n. 12, 2012.

YIN, Robert K. Estudo de caso: planejamento e métodos. 3. ed. Porto Alegre: Bookman, 2005.

Viviane Aparecida Alvares da Silva é mestre em administração pela Faculdade de Ciências Econômicas (Face) da Universidade Federal de Minas Gerais (UFMG). Pesquisadora do Observatório de Recursos Humanos em Saúde da Face/UFMG. E-mail: viviane_alvares@yahoo.com.br.

Allan Claudius Queiroz Barbosa é professor associado da Faculdade de Ciências Econômicas (Face) da Universidade Federal de Minas Gerais (UFMG). Coordenador do Observatório de Recursos Humanos em Saúde da Face/UFMG. E-mail: allan@ufmg.br.

Thiago Augusto Hernandes Rocha é doutorando em administração pela Faculdade de Ciências Econômicas (Face/Cepead) da Universidade Federal de Minas Gerais (UFMG). Pesquisador do Observatório de Recursos Humanos em Saúde da Face/UFMG. E-mail: rochahernandes285@yahoo.com.br 